\title{
Seed Production and Melt Replenishment for the Czochralski Growth of Silicon Germanium
}

\author{
N. ARmour AND S. DOST* \\ Crystal Growth Laboratory, University of Victoria, Victoria, BC, V8W 3P6, Canada
}

\begin{abstract}
The silicon transport in a silicon-germanium melt has been studied to address the issues of melt replenishment and seed production for the Czochralski growth of silicon germanium (SiGe) crystals. The growth of SiGe single crystals by the Czochralski method requires that the melt be replenished with silicon during the growth process due to the rejection of germanium into the melt during solidification. To facilitate the replenishment of the melt, an accurate knowledge of the dissolution rate of silicon into the melt and its transport through the melt is required. To address these issues, a number of experiments have been carried out on the dissolution of silicon into a germanium melt. Liquid phase diffusion growth experiments were also conducted for insight into transport and as a possible method for seed crystal production. The experiments encompassed various temperatures, crucible geometries, crucible translation, and magnetic field levels to determine optimum conditions for the most favorable dissolution rates and mass transport in the melt. Results have shown that replenishment from bottom of the crucible is most effective due to the enhanced silicon transport by buoyancy. The application of magnetic fields may also provide an effective mean to control the replenishment rate (mass transport rate) in the melt.
\end{abstract}

DOI: 10.12693/APhysPolA.124.198

PACS: 61.82.Fk, 81.10.Dn, 66.10.C-

\section{Introduction}

Silicon germanium (SiGe) is an emerging semiconductor material. It possesses full miscibility across its composition range allowing for adjustment of the band gap and lattice parameter [1]. Most importantly, SiGe is compatible with existing silicon technology. This allows for ease of integration into current technology. Producing bulk material is most efficiently done using the Czochralski technique. However, producing compositionally uniform material requires that silicon be replenished in the melt [2].

Czochralski grown SiGe crystals have been produced across the entire composition range. Conventional Czochralski has been used to produce graded composition material [3-7]. The variation in composition is due to the separation between the solidus and liquidus lines. The melt depletes in silicon as SiGe is solidified. The reduction of silicon in the melt changes the composition of the growing crystal as well as reducing the temperature at which solidification takes place. In addition to a graded composition, the growth interface can also be destabilized by the composition changes. These factors render traditional Czochralski methods impossible for quality bulk SiGe material production.

To correct the composition variation, a method to control the composition of the melt is required. These methods tend to fall into two groups, silicon re-feeding and double-crucible techniques (see Fig. 1). The silicon re-feeding techniques introduce additional silicon into the

*corresponding author; e-mail: sdost@me.uvic.ca melt by dissolving solid silicon into the melt by a variety of methods. The simplest method is to lower silicon rods into the top surface of the melt at a rate appropriate to the depletion rate (left in Fig. 1). Other methods, like introducing the silicon at the bottom of the crucible, have also been proposed.
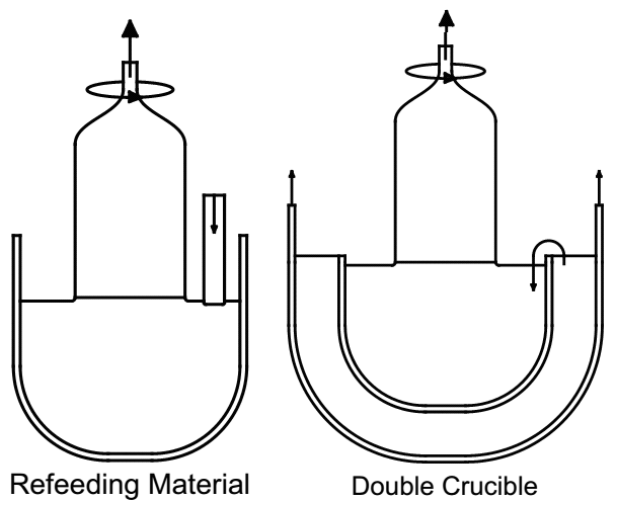

Fig. 1. Schematic views of Czochralski crucibles for replenishment.

In double crucible techniques, there is an inner growth crucible and an encompassing external crucible that contains reservoir material (right in Fig. 1). As a crystal is pulled from the inner crucible, material is transferred from the reservoir to the growth crucible to maintain the growth melt composition. The reservoir material can be introduced to the growth crucible from the bottom or the top. The relative translation rates of the inner and outer crucibles are used to control the material transfer rate.

The success of either of these techniques is tied to how they leverage the mechanisms of transport of silicon 
through a silicon-germanium melt. In order to stabilize the composition and growth interface of the growing crystal, the introduced silicon must replenish the region of the melt depleted in silicon around the growth interface at a rate equal to the depletion rate. In an ideal arrangement, a composition gradient will be present in the melt. The region of replenishment will be at a higher silicon composition than the bulk melt and the area around the growth interface will be at a lower silicon composition. The bulk of the melt, due to convection, will be a relatively stable composition somewhere between the extremes. The mixing rate of the replenishment region into the bulk is matched to the transport of silicon from the bulk into the growth interface region.

To achieve this type arrangement, the mixing in the melt must be strong enough to keep the bulk of the melt at a relatively constant composition. This means that the silicon being replenished must be quickly mixed into the entirety of the bulk. This must be balanced with the need for a stable growth interface for which melt flow should not be too strong. The mechanisms for the incorporation of silicon into the melt are very important for these techniques of growth. The orientation of the replenishment region for instance can be very important due to the strong solutal buoyancy of silicon in a germanium melt.

At the growth interface, the transport for silicon from the bulk to the growth interface is the rate-limiting step for growth and sets the required replenishment rate. For interface stability, flow velocities should be low in this region and therefore the diffusion component of transport will be very important to determining appropriate growth rate.

The re-feeding of silicon into the melt has a distinct advantage in simplicity. However, there is little control of composition of the replenishment region in the melt. This can lead to unfavorable flow conditions in the melt due to the solutal buoyancy of the silicon species. The double-crucible technique is a technically more complex process, but offers the advantage of replenishment region composition control and removal of the dissolution step necessary when re-feeding silicon. Both melt replenishment techniques currently show compositional variations in grown crystals [8].

The availability of SiGe seeds also affects the effectiveness of the Czochralski technique for bulk growth of SiGe. Most studies on the Czochralski growth use Si seeds for the entire SiGe composition range. Ge seeds are also utilized for Ge-rich SiGe [9]. It has been noted that the use of such seeds cause a high density of dislocations at the seed-crystal interface due to the lattice mismatch [10]. In addition, the grown material often features twin defects and transition to polycrystalline growth $[11,12]$.

In order to address the above-mentioned issues in the Czochralski growth, we have carried out a series of dissolution experiments of silicon into germanium. The objectives of these dissolution experiments were to determine accurately the dissolution rate of silicon into germanium and also the diffusion coefficient of silicon in the $\mathrm{Si}-\mathrm{Ge}$ melt. Accurate values of silicon diffusion and dissolution rates are essential for accurate predictions from numerical simulations. We also aimed at determining most optimum crucible stack orientation for an efficient and uniform silicon replenishment. Experiments performed with and without the application of external magnetic fields have shown that replenishment from bottom of the crucible is most effective due to the enhanced silicon transport by buoyancy. The application of a static magnetic field has altered the thermal characteristics of the dissolution process being not so favorable. However, the application of either a rotating magnetic field (RMF) alone or an RMF combined with static field provides better mixing and control of replenishment. Experiments using long capillary samples have shown similar trends.

We have also developed the liquid phase diffusion (LPD) technique to produce SiGe crystals with graded silicon composition. We aimed to grow the needed seed crystals with desired silicon compositions for $\mathrm{Cz}$ growth. Experiments with and without the application of external magnetic fields have shown similar observations obtained in the dissolution experiments, namely, an RMF alone or combined with static field is beneficial, but the application of a static field alone disturbs the stable growth mechanism of LPD. Experiments under a modified thermal profile (with heat sink at bottom) provide a better control for obtaining higher silicon compositions (about $20 \%$ ) in the grown crystals. This implies that in our future LPD experiments under appropriate crucible translation, SiGe seed crystals at desired uniform silicon compositions can be obtained.

Novelty and significance of the experimental results are described in detail at the end of each section.

\section{Dissolution of silicon into germanium melt}

We have first examined the diffusion mechanism of silicon in a germanium melt experimentally [13]. Experiments were specifically devised to examine the effects of the orientation of crucible (effect of the gravity direction) and also free surface. The effect of gravity on silicon transport in crystal growth experiments may be significant due to the large difference between germanium and silicon densities. Silicon, being the lighter component, has a tendency to float in the germanium melt. In order to determine the contribution of gravity experimentally, as explained later in detail, two configurations were considered: one with the silicon seed at top of the germanium melt, and the other one with the seed at the bottom. Two sets of experiments, with and without a free surface, were also designed to show the effect of a free surface on dissolution, namely the effect of surface tension driven Marangoni convection when a free surface exists.

\subsection{Experimental procedure}

The experiments were conducted in a three-zone vertical dc resistance tube furnace under isothermal condi- 
tions, which were maintained over the crucible length at $1100{ }^{\circ} \mathrm{C}$. The materials were contained in a quartz crucible. The silicon used was single crystal optical grade, $5 \mathrm{~N}$, material. The germanium used was $6 \mathrm{~N}$ material. The silicon and germanium were both cleaned and etched prior to loading into the crucible. The cleaning, etching and loading took place in a cleanroom environment. Once the materials were loaded, the crucible was evacuated to approximately $1 \times 10^{-3} \mathrm{~Pa}$. The crucible was then sealed with a hydrogen torch.

The crucible, pictured in Fig. 2, was hung in the furnace on a stainless steel rod. It was first preheated for approximately $1 \mathrm{~h}$ above the hot zone at approximately $800^{\circ} \mathrm{C}$. To start the experiment, the crucible was dropped into the isothermal area of the furnace from the preheating position. It was allowed to remain there for the selected experiment time (durations of 10,20 , or 30 min were selected). At the conclusion of the experimental time, the crucible was pulled from the furnace and quickly quenched in ice-water.

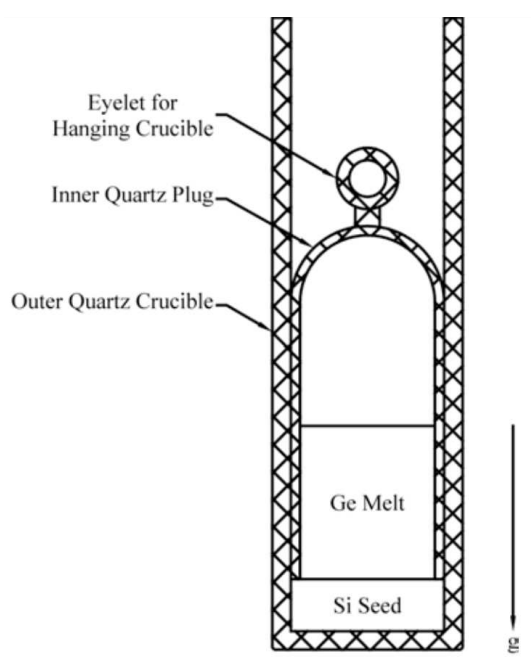

Fig. 2. Crucible configuration for bottom seeded experiments. Top seeded experiments are identical except the seeds sit at top of the Ge melt. Dimensions: sample diameter is $24 \mathrm{~mm}$, silicon seed height is $5 \mathrm{~mm}$, and germanium melt height is $30 \mathrm{~mm}$.

The samples were then sectioned axially into two bulk halves and a $2 \mathrm{~mm}$ thick center slice. All pieces were polished for analysis. One bulk section was differentially etched to reveal structure. The etch used was a 4:1:1, $\mathrm{H}_{2} \mathrm{O}: \mathrm{H}_{2} \mathrm{O}_{2}: \mathrm{HF}$ solution. The center slice was used for energy dispersive spectroscopy (EDS) compositional analysis.

Three different material arrangements were used (Fig. 3). The different configurations are illustrated. The first was a silicon seed secured at the bottom of the crucible with germanium located above. The germanium quickly melts on introduction to the $1100^{\circ} \mathrm{C}$ section of the furnace and begins to dissolve the silicon. This arrangement features a free surface at the top of the germa- nium melt, exposed to vacuum. The second arrangement covers the free surface of the melt. A graphite cap is floated on top of the melt to prevent Marangoni convection. The final arrangement was the silicon seed floating on top of the germanium melt. In this case the silicon seed covers the melt free surface. This arrangement is similar to the crucible stacking used in the liquid phase diffusion (LPD) growth system for SiGe used in Ref. [14].

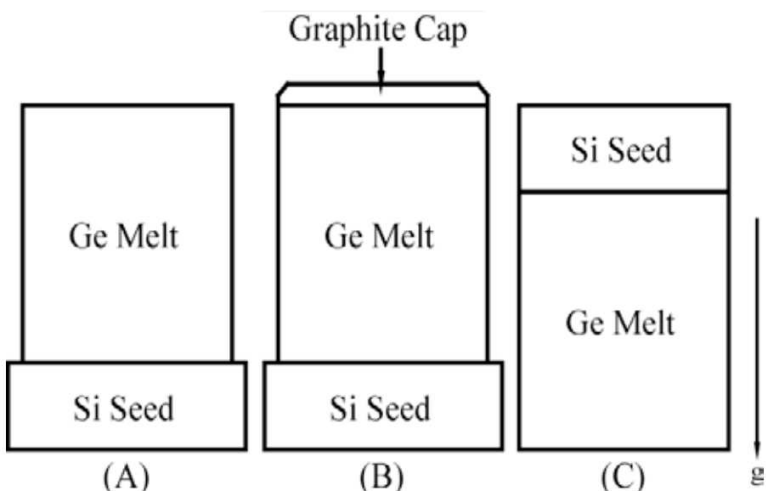

Fig. 3. The three material configurations. Setup A features a free surface on top of the melt. Setup B has the free surface covered. Setup C is the same as setup B with the direction of dissolution reversed.

Initial trial experiments were conducted with the seed secured at the bottom of the system to gauge dissolution rate. These experiments were conducted to determine the appropriate time durations for the study, and as a result, the experiment times of 10,20 , and 30 min were chosen.

The quenching in ice water was performed to maintain the concentration profile developed during the experiment. The fast transition to solid should capture the concentration profile and not allow further diffusion. A scanning electron microscope (SEM) equipped with an EDAX EDS detector was used for determining the concentration profile in the quenched material. The quenching process was very hard on the material resulting in many fractures. To maintain the relative spatial location of all points in the melt, the fractured pieces were joined back into position with epoxy.

\subsection{Results and discussion}

The experiments with the silicon seed on the bottom with the top of the melt a free surface exhibited a high dissolution rate. In these experiments, the dissolution height was in the 2 to $3 \mathrm{~mm}$ range. The time evolution of the dissolution showed a decaying increase trend as seen in Fig. 4. This is likely due to the melt approaching its saturation point. As the melt becomes saturated, it loses its ability to dissolve more silicon, which slows dissolution. This time evolution trend was also observed in the experiments with the same configuration but with the free surface covered (no free surface). 


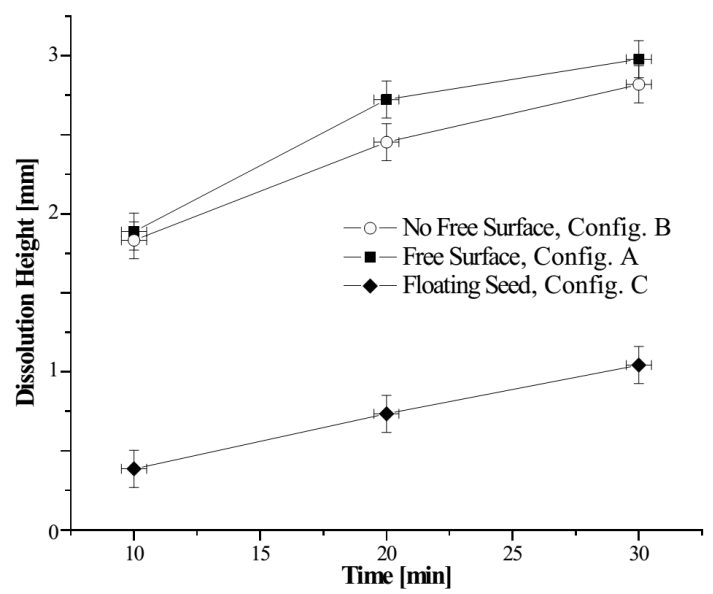

Fig. 4. Plot showing the trends of all three sets of experiments. The linear increasing trend of the floating seed experiments is visible. The actual dissolution height without any gravity effect would be expected somewhere between the values of no free surface (setup B) and floating seed (setup C).

By comparing the dissolution heights of these two experiment configurations, a clear trend is visible. The experiments conducted with a free surface on top of the melt dissolved more silicon than the experiments with a graphite cap on top of the melt. This clearly shows the contribution of the Marangoni convection to the silicon transport. The experiments were conducted under isothermal conditions; therefore radial temperature gradients were minimized. Concentration gradients across the free surface will be present and contribute to the Marangoni convection. The magnitudes of these gradients are unclear from the compositional measurements. Given the tendency of silicon to build up at the free surface due to its buoyancy in the germanium, it would be expected that the concentration gradients would be quite low. In the experimental setups used here the area of the free surface is relatively small when compared to the volume of the melt. The surface area is approximately $380 \mathrm{~mm}^{2}$ and the volume $9500 \mathrm{~mm}^{3}$. It is likely that the effect of surface tension would become magnified as this ratio is adjusted to higher surface area. This could be significant in crystal growth systems using large melts such as Czochralski. The contribution of free surface convection to mixing is substantial enough to warrant attention when considering SiGe growth systems.

The dissolution interface exhibits signs of being unstable when it is at the bottom of the melt. This is very apparent in the 30 min samples. These samples show a very wavy interface. This is likely due to strong solutal convection along the interface, showing the strong effect of gravity. Such an interface can lead to the formation of unstable flow structures in the melt. In Fig. 5, the boundary layer at the interface is visible under $10 \times$ magnification on the differentially etched samples. It shows consistent thickness for all experimental setups and time steps, including the floating silicon seed configuration.

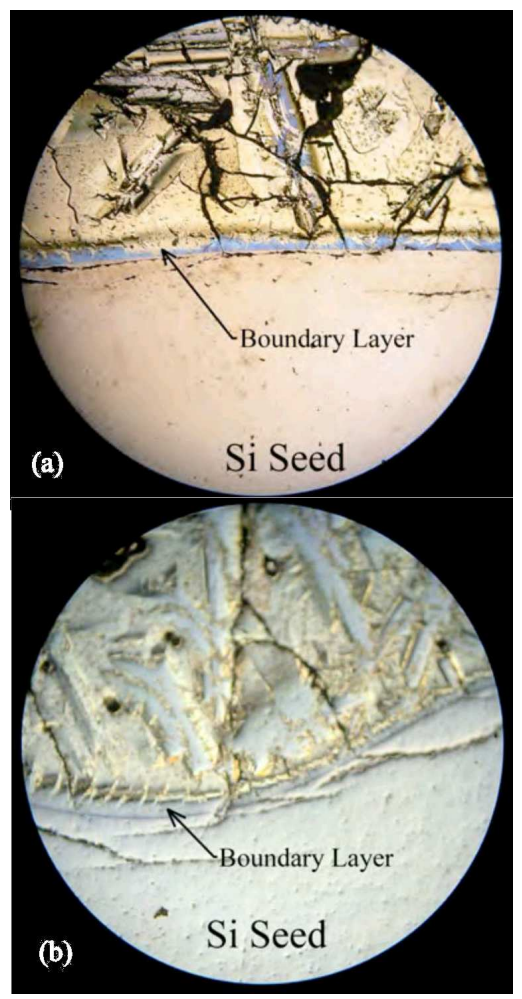

Fig. 5. (a) 10 min free surface sample, setup A. Interface region under $10 \times$ magnification. The boundary layer on the stable interface is indicated. (b) $30 \mathrm{~min}$ free surface sample, setup A. Interface region under $10 \times$ magnification. The boundary layer on the unstable interface is indicated.

The energy dispersive spectroscopy (EDS) measurements performed on both the samples with and without a free surface showed very similar results. The experimental measurements scattered notably. The results from the three experiments conducted with the free surface are plotted in Fig. 6 . This could be due to either the quenching process or the high rate of dissolution and mixing. The time frame for the melt composition to become homogenized and stable may simply be longer than the 30 min time duration examined. The quenching process should be quick enough to avoid macroscopic segregation, but microscopic segregation will have occurred. To mitigate this, a large frame size, $450 \mu \mathrm{m}$ by $450 \mu \mathrm{m}$, was used for the EDS sampling. A trend that did emerge is a drop in silicon concentration through the middle of the melt. This was present in the profiles run down the centerline of the samples and at a $6 \mathrm{~mm}$ offset.

The LPD process, utilized in previous work [14], considers the silicon dissolution from the top of the melt (similar to setup C in Fig. 3). In this apparatus, a silicon source approximately $2 \mathrm{~mm}$ thick will last the duration of the experiment. A typical LPD experiment will last around 5 days. In the bottom seeded experiments, it takes just over $10 \mathrm{~min}$ to dissolve $2 \mathrm{~mm}$ of silicon. This indicates the strong effect of gravity on the dissolution 


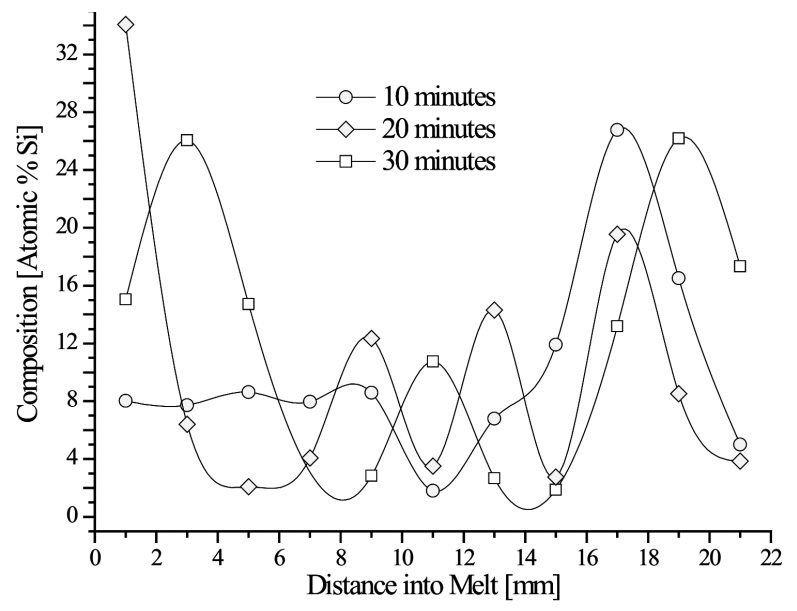

Fig. 6. Plot showing the time evolution of the samples with the top of the melt as a free surface, setup A. The scatter in the measurements is evident. The trend to a lower silicon concentration in the middle of the melt is also visible. The profiles here are from the centerline of the sample.

mechanism. This stems for the high density difference between silicon and germanium. Silicon is substantially less dense than germanium and is therefore buoyant in the melt. With the dissolution interface at the top, the buoyancy of the silicon counteracts its transport downwards into the melt. This phenomenon reduces the contribution of convective flow and causes the transport to become diffusion dominated. Growth therefore proceeds at slow rates. This was tested in a set of experiments with the seed at the top of the melt (setup C in Fig. 3).

The dedicated floating seed experiments used the same periods of time as the other experiments to allow for comparison. The dissolution height was in the 0.5 to $1 \mathrm{~mm}$ range, substantially less than the other configuration. In addition, the amount dissolved through time increases approximately linearly. This is shown in Fig. 7. It does not show the decay behavior indicated in the other experiments. The much lower dissolution rate is delaying the onset of melt saturation. The dissolution interface shows a flat profile. It does not show any of the instability present in the other experiments. The information in Fig. 7 may provide insight into the actual diffusion rate under no gravity. This value will be somewhere between the values of no free surface ( setup B) and floating seed ( $\operatorname{setup} \mathrm{C})$. The penetration of silicon from the dissolution interface into the melt can be easily seen on the differentially etched samples. The area where silicon is present is characterized by a fine needle-like crystal structure. Areas of germanium are indicated by large grains of varying color.

Figure 8 shows the transport of silicon into the melt in the samples of 10, 20, and $30 \mathrm{~min}$. In the $10 \mathrm{~min}$ sample there is very little, while in the 30 min sample the needle structure covers half the sample. The lines on the photos show the upper extent of the needle structure.
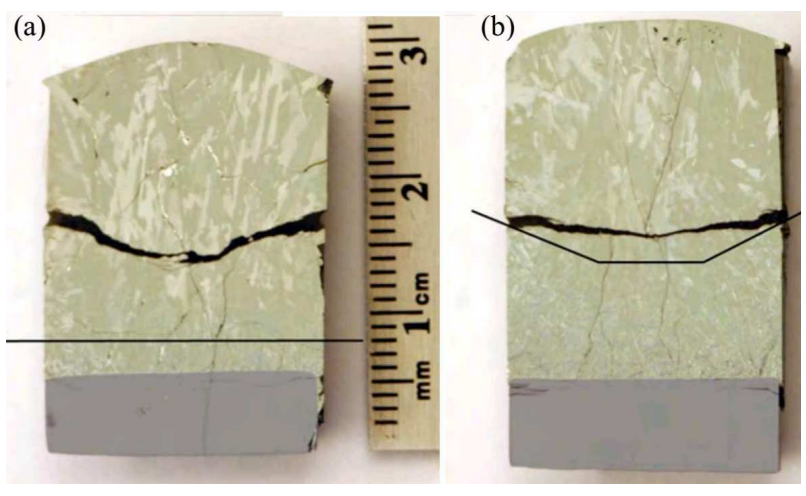

Fig. 7. (a) The floating seed experiment run for 10 min. The stable interface is visible. (b) The floating seed experiment run for $30 \mathrm{~min}$. The interface shows no sign of instability. The lines in the picture indicate the extent of the needle structure grains associated with the presence of silicon. Large germanium grains are visible above this line. The samples were differentially etched to expose the structure. The visible difference in heights of the material is mainly due to sectioning orientation and variations in the quartz crucibles used. Similar Si seed heights and weights of Ge were used in all experiments.

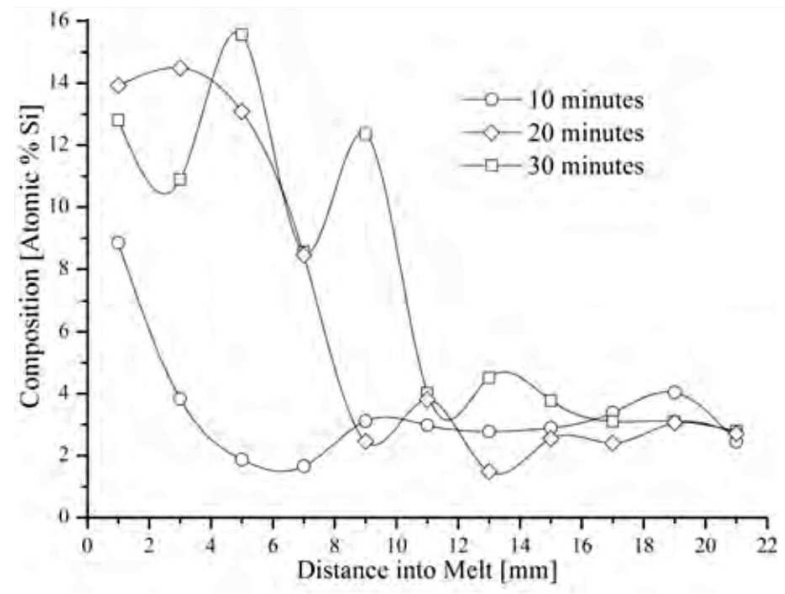

Fig. 8. Plot of the EDS measurements in the floating seed experiments. The progress of the silicon into the melt is evident. The composition measurements were taken from the centerline of the sample.

When considering growth systems for SiGe, the separation between the liquidus and solidus lines is of prime concern. Growing this material from the melt often utilizes melt replenishment techniques. In these cases, more silicon is introduced into the melt to prevent it from becoming germanium rich. As this addition is localized in the melt, it must be transported quickly to the growth interface to be effective. When considering where to add the replenishment material, it is necessary to consider the buoyant effect of the silicon. The silicon should be introduced in such a way as to utilize the buoyant effect to allow for the fastest possible silicon transport. Re- 
plenishing the melt from above, as is often the case, may result in slow transport of the silicon to other areas of the melt.

The LPD growth process mentioned earlier has been successful at producing single crystal materials. One of disadvantages of the system is that it is driven by diffusion only. This makes growth very slow. In the vertical arrangement, the diffusion process is additionally slowed by the buoyant silicon. It is possible that the growth rate could be improved on by using the silicon buoyancy to aid diffusion.

\subsection{Remarks}

The results clearly indicate a much higher level of mixing in the bottom-seeded samples. The wide scatter in the compositional measurements would seem to show the effects of macrosegregation due to the wide spacing of the solidus and liquidus lines. While the free surface produced only a small change in the dissolved height of the silicon seed, it did show a trend to increased dissolution at all experiment times. The experimental time frames were sufficient to see the slow down of dissolution as the melt begins to reach saturation. Instability of the interface due to the high convective flows is evident in the bottom-seeded samples.

The change from bottom seeded to top seeded, effectively changing the direction of gravity, drastically altered the rate of silicon transport. The top seeded experiments proceeded diffusion like when compared with the high convection evident in the bottom-seeded samples. The much slower transport should raise questions about the effectiveness of replenishing the melt in Czochralski from above. A more efficient way may be introducing the silicon at the bottom beneath the growth interface. This could quickly raise silicon concentration at the growth interface. With the growth interface at the top of the melt, it should be less subject to compositional fluctuations, as the silicon will tend to float on top of the melt. The drawback will be a strong flow field due to the strong solutal buoyancy. These strong currents could destabilize the growth interface.

The LPD growth process may be aided by a geometry change to utilize the buoyancy effect of the silicon [14]. The faster transport would drastically increase growth rate. It is possible that the strong natural convection flows could be controlled through a static magnetic field. This could allow stable growth to proceed. Other similar solution techniques like travelling heater method (THM) could also potentially benefit from a geometry reevaluation [15].

\section{Effect of a static magnetic field on the dissolution process}

As described in the previous section, the buoyancy of the silicon in the melt was found to have profound consequences on the dissolution rate and mixing of silicon into the germanium. The questions then were (a) whether the convection in the melt was enhancing or reducing the dissolution of silicon in this system, and (b) whether stable growth can be achieved under an applied static magnetic field (under altered convection conditions in the melt). In order to address these questions, a vertical static magnetic field was applied during the experiments in this study to observe the dissolution and mixing processes of silicon in the germanium melt.

\subsection{Experimental procedure}

The experimental procedure was the same as described in Sect. 2. The experimental setup was kept identical in order to properly compare results. This time the furnace was placed inside the bore of $1.25 \mathrm{~T}$ superconducting magnet. The vertical magnetic field chosen for these experiments was $0.8 \mathrm{~T}$. This field strength was chosen based upon previous numerical work using the magnetic field to suppress convection [16, 17], and was considered sufficient to provide the required suppression in the $\mathrm{Ge}$ melt. The crucible, when in the $1100{ }^{\circ} \mathrm{C}$ section of the furnace, is located in the most uniform section of the field. The magnetic field was kept on during the entire experimental process.

The crucible setups (A) and (B) are used. The basic setup is a silicon seed secured at the bottom of the crucible with the germanium located above. The germanium quickly melts on introduction to the $1100^{\circ} \mathrm{C}$ section of the furnace and begins to dissolve the silicon. The first arrangement features a free surface at the top of the germanium melt, exposed to vacuum. The second arrangement covers the free surface of the melt by means of a graphite cap floating on top of the melt, to eliminate the effect of free surface on the flow structure of the melt.

\subsection{Results and discussions}

The measured dissolution heights in the samples are shown in Fig. 9. As seen, the comparison of the samples with the free surface to the samples with the surface covered shows that the dissolution heights are higher in the samples with the presence of a free surface, which is the same trend observed in the experiments conducted under no magnetic field [13]. This means that the application of a static magnetic field in this experimental system did not have a significant effect (within the limit of measurement errors) on the contribution of free surface to the dissolution process. As we will discuss it later in detail, one may think that the flow structure of the melt in both cases are altered significantly in the presence of magnetic field, leading to higher dissolution heights in both cases. The effect of magnetic field on the flow structure in the melt (both the gravity- and surface tension-driven convective flows), and the relative contributions of these flows to the dissolution process can only be determined through a full fluid flow analysis including thermal and solutal effects [18, 19].

Comparing the experiments conducted under magnetic field to those with no applied field, one can see that there is a trend to higher dissolution with the application of 


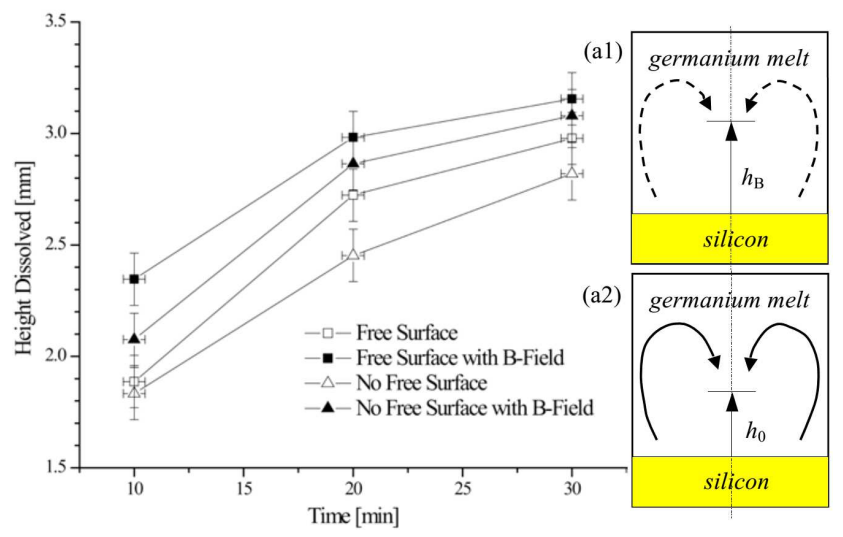

Fig. 9. The dissolved height of silicon versus experiment time (on left). The experiments without magnetic field presented here were performed under the identical conditions of Ref. [13] other than the applied magnetic field. The trend to higher silicon dissolution in the presence of the magnetic field is clearly visible. (a) A schematic representation of flow cells in the melt: (a1) under the effect of magnetic field, and (a2) in the absence of magnetic field. $h_{\mathrm{B}}$ and $h_{0}$ describe symbolically the dissolution heights in (a1) and (a2), respectively.

the magnetic field, as illustrated in Fig. 9a. In other words, the application of a static magnetic field (under the altered convection condition in the melt) enhanced the dissolution of silicon into the melt in both setups.

This result can be explained in setup B as follows. As mentioned earlier, the dissolution experiment was initiated by lowering the crucible from $800{ }^{\circ} \mathrm{C}$ to the region of $1100{ }^{\circ} \mathrm{C}$ in the furnace. As seen from the thermal analysis [20], the outer region of the melt remains hotter than the inside until the melt reaches a thermal steady-state. Therefore, it is estimated intuitively that the thermal characteristic of the present system (melt being hotter near the crucible vertical wall) leads to a convective flow in the melt with two convection cells (symbolic representation of natural convection) circulating upward along the wall and downward in the centre (just opposite to what was observed in the LPD growth of SiGe in $[14,16,17])$, as shown schematically in Fig. 9b. These convection cells slow the dissolution of the silicon into the melt by remixing dissolved silicon towards the dissolution interface reducing the concentration gradient. This causes the observed lower dissolution height.

The application of the magnetic field changes the flow structure and prohibits the formation of large convection cells in the melt (Fig. 9b). This reduces mixing of the silicon into the germanium. As a result, the buoyancy of the silicon in the germanium melt transports it quickly away from the interface and it tends to build up at the top of the melt. Without the applied magnetic field, strong convection cells would remix this silicon from the top of the melt back into the body of the melt and towards the dissolution interface. Considering the concentration gradient around the interface, a higher gradient will naturally lead to a higher rate of dissolution. The remixing of silicon back towards the interface will slow dissolution by reducing the concentration gradient at the interface. Application of the static magnetic field in this system prevents the remixing and leads to higher dissolution rates.

One can also make similar statements for setup A since the same trend in dissolution of silicon into the melt was observed. This indicates that the altered flow structure in the melt under the applied magnetic field is responsible for the higher dissolution heights in this system. However, it is not possible to make a quantitative comment on the relative contributions of natural and Marangoni convections without a full numerical fluid flow analysis for the melt. A comparison of the solutal and thermal Grashof numbers shows the relative contribution of thermal and solutal buoyancy to the system compared with viscous forces. The high solutal Grashof number for this system indicates the importance of the solutal component of the buoyancy over the thermal component. The definition of these numbers and their values are presented respectively as

$$
\begin{aligned}
& \mathrm{Gr}_{\mathrm{T}}=\frac{\beta_{\mathrm{T}} g l_{0}^{3} \Delta T}{\nu^{2}}=1.08 \times 10^{7} \\
& \text { and } \operatorname{Gr}_{\mathrm{c}}=\frac{\beta_{\mathrm{c}} g l_{0}^{3} \Delta c}{\nu^{2}}=1.91 \times 10^{7},
\end{aligned}
$$

where $l_{0}$ is the solution height, $\nu$ - the kinematic viscosity, $g$ - the gravitational constant, and $\beta_{\mathrm{T}}$ and $\beta_{\mathrm{c}}$ represent the thermal and solutal expansion coefficients, respectively.

The silicon concentration in the melt has become inhomogeneous due to the altered flow structure under the magnetic field. This effect can be seen macroscopically in the quenched material (Fig. 10). Areas where silicon is present in the germanium melt are characterized by a fine needle-like structure. This structure is prominent close to the interface and near the top and sides of the melt. However, the center region of the melt shows a noticeable reduction of this structure (Fig. 10a). This would seem to indicate a lower concentration of silicon in the center region. The composition profiles, as measured with EDS, support the trend of a lower silicon concentration in the center of the melt [20].

In the experiments conducted without the applied magnetic field, the dissolution interface showed evidence of flow instability. This was indicated by a wavy dissolution interface. Application of the magnetic field should reduce the radial flow in the melt and therefore reduce flow instability along the interface. With application of the magnetic field, the interface did show greatly reduced signs of instability. This result is shown in Fig. 11.

While the interface shows signs of improved stability, the concentration profiles obtained from the EDS results continued to show a high degree of scatter [20]. This scatter was also present in the samples without the applied field. The cause of the scatter is not definite. Segregation of the silicon from the germanium during the quenching process is a likely candidate. It may also be that the melt 

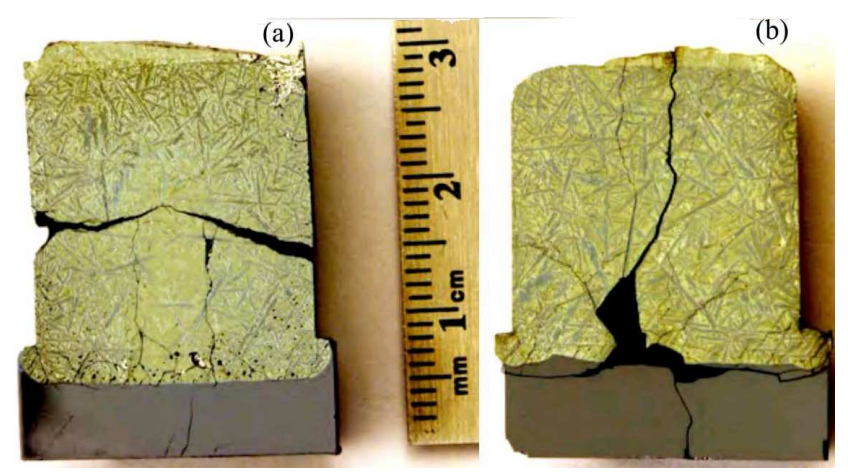

Fig. 10. Pictures of the needle structure in the quenched samples. Both samples are for an experiment time of $20 \mathrm{~min}$ and the free surface was covered (configuration B). The samples were differentially etched to reveal the needle structure. (a) Sample from experiment conducted with magnetic field. (b) Sample from experiment conducted without magnetic field. The silicon depleted center region is visible in the sample on the left where there is a lack of structure.

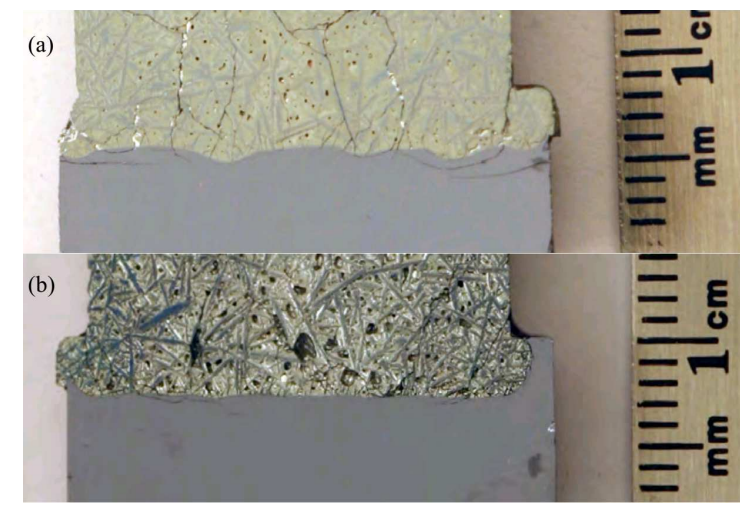

Fig. 11. Pictures of interface stability in experiments conducted with free surface (configuration A): (a) $30 \mathrm{~min}$ sample with no applied field, and (b) $30 \mathrm{~min}$ sample under magnetic field.

has not had a chance to homogenize given the relatively short duration of the experiments.

\subsection{Remarks}

The experimental results of the present setups show that the silicon dissolution was enhanced under an applied magnetic field. This observation can be attributed to the altered flow structure in the melt. The result indicates that the convection in the germanium melt without the application of magnetic field [13] was reducing the dissolution rate of silicon. One may then conclude that the benefit (or effectiveness) of an applied magnetic field in a system is related to the flow characteristics of the melt. By comparing these results to previous work carried out without magnetic field, we observe that the change in the dissolution behavior of the silicon is obvious, and although the application of magnetic field leads to a more stable dissolution interface, it may still not be sufficient to make the interface as stable as the one when the silicon source is at the top. The influence of the free surface on mixing remained unchanged with the application of the magnetic field. There is a trend to higher dissolution rates with the presence of a free surface on the melt.

\section{Diffusion limited silicon dissolution into germanium melt}

In previous sections, we have shown that dissolution has been influenced by flow structure in the germanium melt [13]. The dissolving silicon stabilizes the melt against convection in the configuration where silicon is dissolving into the melt from above (setup C in Fig. 3). This is due to the lighter silicon reducing the density of the melt as the silicon concentration increases. This helps to weaken any convective flow structure. However, we have seen that the weak flow structure present still has an influence on dissolution. In order to explore the diffusion behavior of silicon in the melt, we have reduced the diameter of the crucibles to $8 \mathrm{~mm}$ in order to further weaken the flow structure in the melt [21].

\subsection{Experimental procedure}

The experimental setup was the same as before. An isotherm was maintained over the crucible length at temperatures of $1000^{\circ} \mathrm{C}, 1050^{\circ} \mathrm{C}$, or $1100^{\circ} \mathrm{C}$. The isotherm is maintained within 3 degrees axially and 1 degree radially. The ampoule was first preheated for approximately $1 \mathrm{~h}$ above the hot zone at approximately $800^{\circ} \mathrm{C}$, i.e. below the melting point of Ge. This quickens the time to melt the sample and begin dissolution into the melt. To start the experiment, the crucible was dropped into the isothermal area of the furnace from the preheating position. It was allowed to remain there for the experiment time, $60 \mathrm{~min}$. At the conclusion of this time, the crucible was pulled from the furnace and allowed to cool in air. The samples were then sectioned into half. One section was differentially etched to reveal structure. Two different material arrangements were used again (B) and (C). Setup $\mathrm{C}$ was used for the majority of experiments. For comparison, two samples were processed at $1050{ }^{\circ} \mathrm{C}$ with the silicon source at the bottom of the crucible B.

\subsection{Results and discussion}

The samples processed with the silicon dissolving from the top all exhibited almost the same level of dissolution over the temperatures used. A dissolution height of around $0.5 \mathrm{~mm}$ was measured across all samples processed as seen in Fig. 12. This implies that the temperature dependence of the dissolution rate and consequently the diffusion coefficient is not overly significant in this range of temperatures. It would appear from results that there is a temperature dependence but it is not resolvable with this experimental setup. The length of experiments 


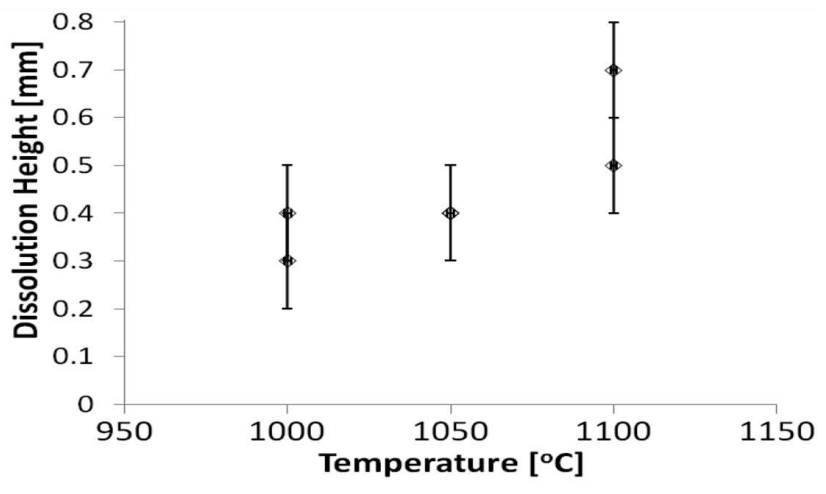

Fig. 12. Plot of dissolution height of processed samples versus temperature. The lack of differential between temperatures with respect to error is apparent.

was one hour. It is possible that a longer experiment time could allow the dependence to be better resolved.

In previous sections, these types of experiments were conducted in a $25 \mathrm{~mm}$ diameter crucible and were run for shorter periods of time and only at $1100^{\circ} \mathrm{C}$. In those experiments, the dissolution height was up to $1 \mathrm{~mm}$. This highlights the increased mixing present in the melt with the larger diameter due to the contribution of stronger convection in the melt [20]. The decreased diameter crucibles used in the current experiment work do help to weaken convection in the melt. Experiments on $4 \mathrm{~mm}$ and $2 \mathrm{~mm}$ diameter crucibles were performed with little success as surface tension often prevented the silicon from fully wetting with the melt and voids formed separating the melt into segments. The crucible would require modification so pressure could be exerted to ensure proper wetting between all elements.

Numerical modelling did not show significant change in flow structure between the $8 \mathrm{~mm}$ and smaller diameter crucibles. As seen in Fig. 13, the composition profiles after the one hour experiment time are virtually identical. The flow structure was computed with the effects of solutal buoyancy and similar weak flow is present in both crucibles. All numerical work was completed using the commercial ANSYS CFX package.

The samples processed with the silicon at the bottom of the melt exhibited a drastically different result. In the cases of a 10 and 30 min experiment times, the silicon source material is completely dissolved. This is dissolution of $5 \mathrm{~mm}$ of silicon. No silicon is visible in the sample with the silicon dissolving from the bottom while most of the seed remains when the silicon is dissolving from the top [21]. This is dramatically faster transport than what was seen in the opposite orientation of the dissolution interface. This highlights the significant effect of gravity (convection) on the dissolution process.

In experiments with growth crucible size ampoules (setups $\mathrm{A}, \mathrm{B}$, and $\mathrm{C}$ ), a difference in transport rates between orientations was observed similar to that seen here but not as dramatic. A key difference in this set of experi-

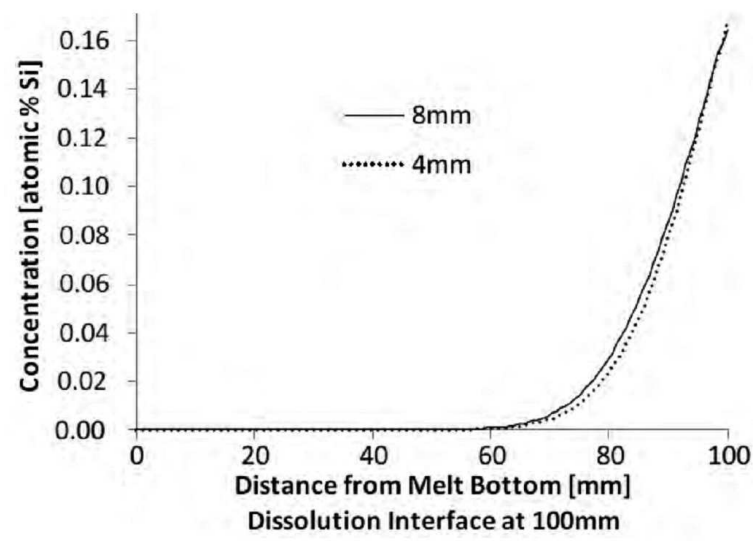

Fig. 13. Computed concentration profiles for dissolution experiments. The solid line is for an $8 \mathrm{~mm}$ diameter crucible and the dotted line is for a $4 \mathrm{~mm}$ diameter crucible. Little difference in the computed concentration profiles is visible. The experiment conditions are $1000^{\circ} \mathrm{C}$ for one hour. The diffusion coefficient was $2.8 \times 10^{-8} \mathrm{~m}^{2} / \mathrm{s}$.

ments is that the ratio of germanium to silicon was increased. Therefore from the previous work, the dissolution of silicon from the bottom of the melt was limited by the saturation of the germanium melt with silicon. The rate at which silicon dissolves in this configuration seems limited only by the saturation level of the melt. Enough silicon is used in the experiment to fully saturate the melt. The approximate composition of the melt at saturation at $1000^{\circ} \mathrm{C}$ is $18 \%$ silicon by mole. As the melt approaches this threshold, the transport away from the dissolution interface will slow and therefore the dissolution rate will slow.

The longer column of germanium was used so that useful composition information could be later extracted to directly estimate the diffusion coefficient. However, due to the low concentration of silicon present in the melt there is significant expansion of the melt during solidification. The presence of silicon in the melt reduces the amount the melt expands on solidification. Due to this expansion during quenching, the melt column is significantly disturbed by cracking and leaking. This, unfortunately, makes it impossible to reliably correlate the position in the column to a composition. The use of shear cell would seem necessary to extract this type of information accurately.

\subsection{Remarks}

Dissolving silicon into a germanium melt in an $8 \mathrm{~mm}$ crucible resulted in a more diffusion-dominated process than previously observed. However, we were unable to observe any temperature dependence of the dissolution height. This indicates that the dissolution rate and therefore the diffusion coefficient are not significantly affected by temperature over the range of temperatures examined here. In addition, it was noted that the flow structure 
with the silicon dissolving from the bottom is extremely strong leading to extremely fast dissolution when compared to the opposite orientation.

\section{Silicon transport under rotating and combined magnetic fields in liquid phase diffusion growth of SiGe seed crystals}

As mentioned in earlier sections, the production of bulk SiGe single crystals by Czochralski requires seed crystals of desired compositions. To address this issue we have utilized a solution growth technique called LPD, and grown $\mathrm{SiGe}$ seed single crystals from the germanium side with varying silicon compositions up to $16 \%$ (for detail, see $[14,16,17,22-26])$. In this section, we want to examine the effects of applied magnetic fields on silicon transport in the SiGe growth melt.

LPD at its stationary mode produces graded bulk SiGe crystals with varying silicon composition along the growth direction, from which wafers with desired silicon composition can be extracted to be used as seeds for the Czochralski growth and also for subsequent growth with uniform crystal composition by other solution growth methods $[14,16]$. The LPD system can also be used as a single process to produce SiGe crystal with uniform axial silicon composition. Once the desired silicon composition is achieved at the stationary mode, at that time the LPD crucible (or the heater) can be translated at a speed matching the mass transport rate of silicon in the melt to produce single crystal $\mathrm{SiGe}$ with uniform $\mathrm{Si}$ composition for the rest of the growth cycle [22].

In the LPD growth ampoule, a Ge single crystal seed is placed at the bottom, polycrystalline $\mathrm{Ge}$ in the middle, and a Si source at the top. A temperature profile (with temperature gradient) is applied along the crucible such that the Ge seed at the bottom remains solid (allowing for some melt back for a successful start), Ge in the middle melts completely (forming the initial solvent or melt), and the Si source at the top remains solid. The $\mathrm{Si}-\mathrm{Ge}$ dissolution interface at the top of the melt is at a higher temperature than the melting point of Ge, and the growth interface (the interface between the Ge-seed and the Ge-rich melt) at the bottom is kept initially at the melting temperature of Ge. Under the effect of the applied temperature profile, according to the SiGe phase diagram, the silicon source dissolves at the hotter dissolution interface into the Ge-melt. The dissolution of Si into Ge creates a concentration gradient across the melt. Diffusion transports Si from high to low concentration and will result in supersaturation of the melt with Si near the growth interface. The supersaturated mixture of $\mathrm{Si}-\mathrm{Ge}$ is then precipitated on the seed at a composition in accordance to the solidus line at the temperature around the growth interface. This LPD setup is illustrated in Fig. 14.

In the melt, the dissolving lighter $\mathrm{Si}$ species diffuses into the heavier Ge-rich melt and sets a concentration gradient of Si through the melt with a decreasing axial silicon concentration towards the growth interface. This

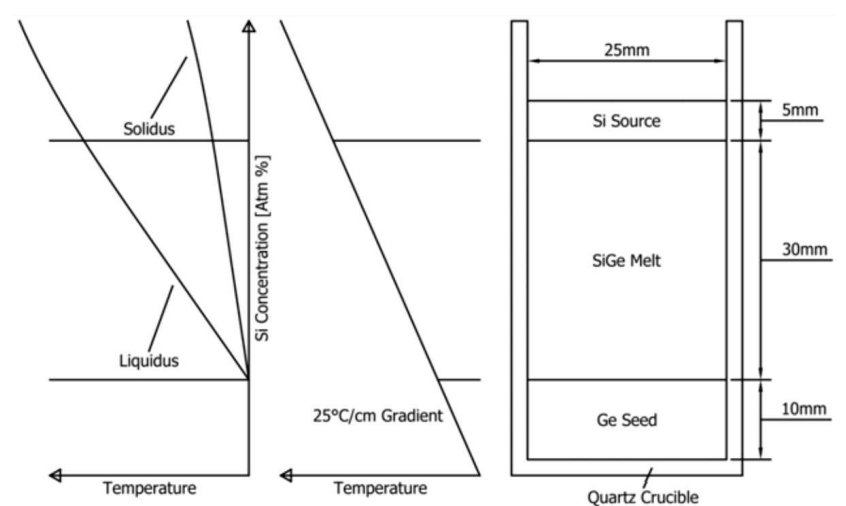

Fig. 14. Far right is a schematic of the LPD crucible. The center plot is the temperature profile applied to the crucible. The left plot is the schematic SiGe phase diagram.

concentration gradient makes the melt buoyantly stable against natural convection and minimizes the effect of natural convection during growth. Convection is strong only near the growth interface during the initial period of growth, but remains weak during the entire LPD process leading to an almost diffusion-dominated but slow growth [14]. However, uniform silicon transport across the melt is essential for radially uniform silicon composition. Our previous numerical simulations (based on the furnace of [14]) have suggested that applied magnetic fields may lead to more uniform silicon transport across the melt, and consequently a flatter growth interface $[16,17]$. In order to examine the effect of applied magnetic fields, we previously performed a set of LPD experiments under an applied vertical static magnetic field [25]. These experiments did not produce the expected improvements; instead, led to significantly reduced silicon transport in the melt. The applied field also disrupted the thermal field of the system to the extent that there was no single crystal growth. The conclusion was that under the experimental conditions (at the $0.4 \mathrm{~T}$ field level) used, the application of a static vertical magnetic field alone was not so beneficial in this LPD system.

In order to enhance silicon transport for faster growth, the addition of a rotating field to induce controlled mixing was proposed. In order to investigate this, we have performed LPD experiments under rotating and combined rotating/static magnetic fields using a three-zone dc furnace system. A series of LPD experiments were performed under the influence of a $5 \mathrm{mT}$ rotating magnetic field at $40 \mathrm{~Hz}$, and also under a combined magnetic field (at a $5 \mathrm{mT}$ rotating magnetic field at $40 \mathrm{~Hz}$, and a $0.4 \mathrm{~T}$ static vertical field). The examination of processed samples show that in both cases silicon transport is enhanced across the melt. The applied rotating magnetic field also seems to lead to flatter growth interface.

\subsection{Experimental setup and procedure}

LPD experiments were conducted using a three-zone dc furnace with a $63 \mathrm{~mm}$ outer diameter bore. The LPD 
furnace was placed inside the bore of a superconducting magnet (is of a $330 \mathrm{~mm}$ bore diameter and generates a vertical static field) such that the crucible vertical axis was aligned with the magnetic field. A six-pole electromagnet array (the rotating magnetic field generator jacket) is placed around the furnace inside the static magnet bore such that it is aligned horizontally with the molten zone in the crucible ampoule. This array generated the $5 \mathrm{mT}$ field rotating at $40 \mathrm{~Hz}$ during the LPD growth. During the experiments under only rotating field, the static magnet was kept off. A $0.4 \mathrm{~T}$ static field was put on for two of the LPD experiments conducted with rotating field (to generate the combined field).

The LPD system is under vacuum in a sealed quartz ampoule at a pressure of $1 \mathrm{MPa}$. The crucible is placed in a temperature gradient of $25 \mathrm{~K} / \mathrm{cm}$. The crucible is $25 \mathrm{~mm}$ in diameter and is loaded with a $10 \mathrm{~mm}$ thick single crystal Ge seed, $30 \mathrm{~mm}$ block of polycrystalline Ge, and a $5 \mathrm{~mm}$ thick single crystal Si source. The crucible is positioned, when the magnetic fields were off, such that the seed is partially melted back when the final temperature profile is reached. The experiments were kept under this applied temperature profile for $72 \mathrm{~h}$ (the arrangements are shown in Figs. 14 and 15).

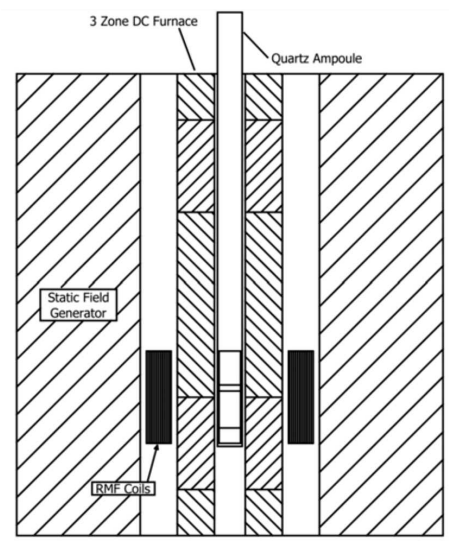

Fig. 15. Schematic of LPD system with the magnetic field generators. The furnace and rotating magnetic field coils are both placed inside the bore of the superconducting magnet used to generate the static field. Dimensions of the systems were given in the text.

A field strength of $5 \mathrm{mT}$ at $40 \mathrm{~Hz}$ was selected based on the results of earlier numerical simulations [17]. Two experiments were conducted with the rotating magnetic field only (static field was off), and two experiments were conducted under combined fields (with the $0.4 \mathrm{~T}$ magnetic field applied in addition to the rotating magnetic field). In order to be able to compare the experimental results and to see the effects of applied magnetic fields, the present experiments were conducted under the same conditions (i.e., applied temperature profile, the location of the growth ampoule, and the material stack in the ampoule were kept the same) and in an identical manner to those conducted previously with no field and under static field only [25].

Silicon composition of the samples (that were sectioned and polished) was determined by EDS analysis. A second section was also differentially etched to show the crystal structure. EDS analysis was performed on a Hitachi SEM equipped with a Oxford EDS system.

\subsection{Results and discussion}

In the samples processed under the RMF alone, there is some seed crystal remaining at the bottom of the melt, but it did fully melt back in the center. The single crystal growth from the edges of the crucible preferentially grew, inhibiting the polycrystalline growth started in the middle of the melt. This can be seen as the triangle of polycrystalline structure at the bottom of the ingot. The two areas of single crystal on either side come from the remaining seed crystal. The limit of growth is identified by a clearly notable striation in the crystal, and the material above this point is the frozen melt during cooling (freezing post experiment). The structure of the frozen melt section is consistent with that of previous experiments $[25,26]$.

The concentration of the samples processed under the RMF show enhanced silicon transport when compared with the samples processed with no field. The increased mixing under RMF has elevated the transport in a consistent manner. The concentration profile maintains the same shape, but shifts the profile upwards to higher silicon concentration for a given distance into the melt. This can be seen in Fig. 16 .

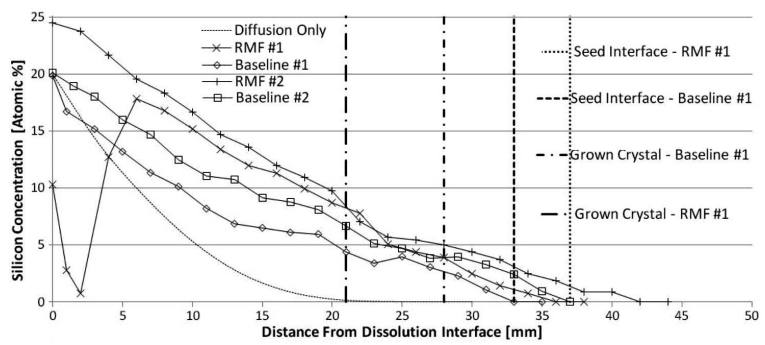

Fig. 16. Comparison of silicon concentration profiles of experiments conducted with a rotating magnetic field and those conducted with no applied magnetic field.

The radial concentration profiles of the RMF samples maintain the relatively flat profile of the baseline samples [26]. The radial concentration profile is not affected significantly by the rotating magnetic field although it leads to slightly more uniform radial compositions compared with those with no field.

From the samples processed under the combined magnetic field, we see that the application of combined fields has changed the thermal field of the system dramatically. This change in the thermal field melted back the seed completely. It appears that the growth was initiated by self-seeding on the bottom of the crucible resulting in a 
fine-grained structure. This is supported by the concentration profiles which show a slight upturn in concentration where growth begins, as seen in Fig. 17. This is indicative of over-saturation of the melt due to lack of nucleation site. The combined field exhibits a very similar concentration profile to that of the rotating field. It does not however bear any similarity to the concentration profile of the experiments under the static field alone, which are presented together in Fig. 18 for comparison.

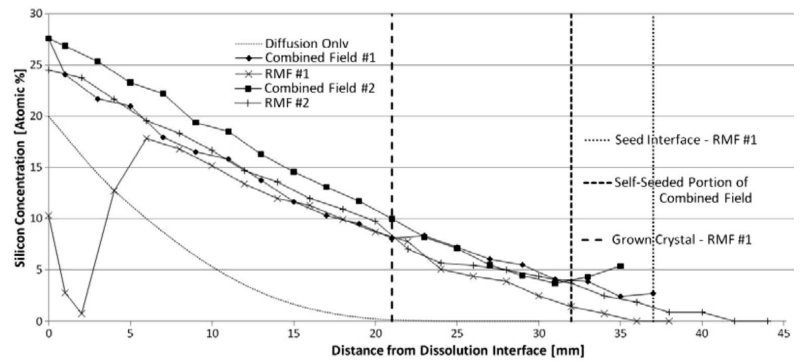

Fig. 17. Concentration profiles of samples processed under rotating magnetic field. One set of samples was additionally processed with the static magnetic field.

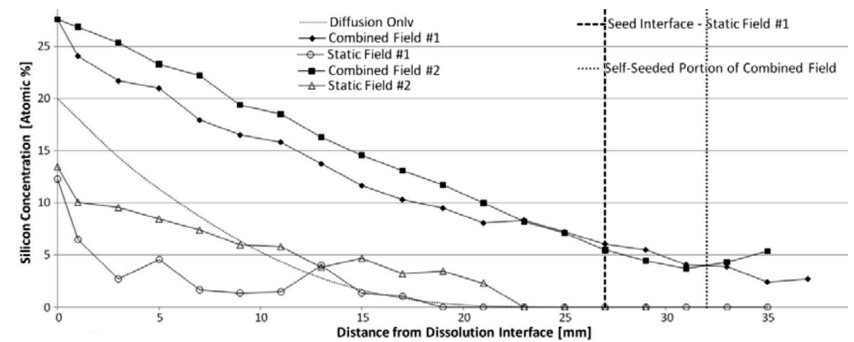

Fig. 18. Concentration profiles of samples processed under applied static magnetic field. One set of samples was additionally processed with the rotating magnetic field.

Comparison of results presented in Fig. 18 may lead to the following conclusions. The present LPD system is mostly stabilized against natural convection by the buoyancy of the silicon in the heavier Ge melt. This weak convection is further suppressed with the application of the static field alone, and the silicon transport in the melt is reduced significantly.

The application of a rotating magnetic field alone, on the other hand, enhances silicon transport by introducing additional mixing into the melt. This additional mixing induced by the rotating magnetic field slightly increases silicon transport in the LPD system.

However, when the static magnetic field is put on together with the rotating field, the effect of RMF is further enhanced since the static field suppresses further the week natural convection in the melt. This is evidenced from the increased transport in the samples processed under the combined field over the samples processed with the rotating field alone.
In most systems the application of combined magnetic fields may provide the most benefit by combining the effects of suppression of convection caused by the applied static field and the additional mixing provided by the rotating field. However, in the present LPD growth system, where natural convection is weak in the melt, it appears that the application of RMF alone offers the most benefit in terms of silicon transport and composition uniformity.

Having said that it would still be beneficial to study the effect of combined rotating/static magnetic field (at various field intensity and frequency levels) in LPD by determining the required applied temperature profile and the crucible location under the fields for a successful single crystal growth. This will require significant thermal profiling using dummy systems under various field levels. We hope to do this in the future.

\subsection{Remarks}

The LPD growth system examined here showed increased silicon transport with the application of a rotating magnetic field. Although this effect was slightly enhanced when a static field was applied at the same time, a complete melt back of the seeds occurred leading to a complete polycrystalline growth. It appears that the application of RMF alone is most beneficial in the LPD system used here. The enhanced silicon transport under RMF could lead to higher growth rates in LPD.

\section{LPD growth with modified thermal profile}

Using exactly the same LPD experimental setup (shown in Figs. 2 and 3), we have conducted LPD growth experiments with a modified thermal field. Schematic of the growth ampoule is shown in Fig. 19. The modified thermal profile in the crucible was envisioned as a method of controlling the growth interface shape. However, the resultant material from these experiments showed that the thermal profile was modified such that much higher than usual Si compositions were reached with the LPD process.

The thermal field was modified by inserting a graphite block underneath the seed crystal. The shape of the block was such that heat extraction from the centre of the growth interface was encouraged. The addition of the graphite had the effect of increasing the overall temperature in the system allowing the LPD process to produce SiGe with higher than usual Si compositions.

\subsection{Results and discussion}

The samples processed with the heat sink in place produced dramatically different results from what has been seen in other experiments. The samples are shown in Fig. 20. The change is likely attributable to a significant rise in the temperature at the bottom of the crucible. The silicon source is completely dissolved in one sample and almost totally consumed in the other. This indicates a substantial increase in the transport in the system. The other notable change is that sample is divided into a top 

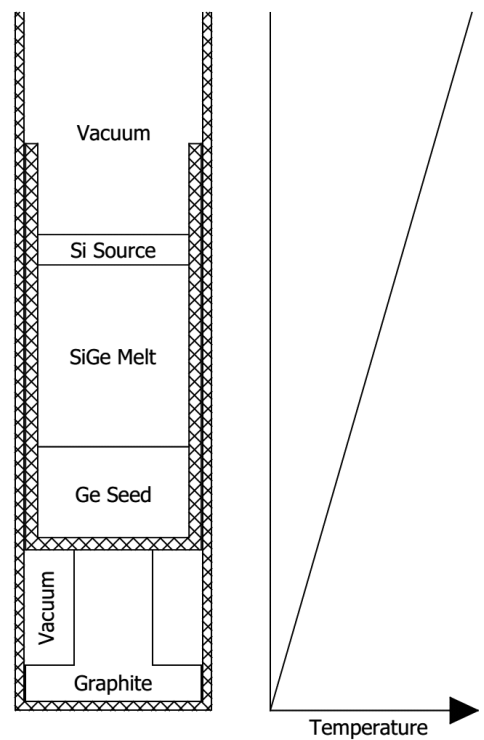

Fig. 19. Schematics of the growth crucible with heat sink.

and bottom section by a distinct abrupt colour change in the material. Near the bottom of the crucible the material is a dark polycrystalline material. At the top near the dissolution interface, the material is much lighter in colour and shows evidence of macro-segregation of silicon in the form of needle-like crystals.
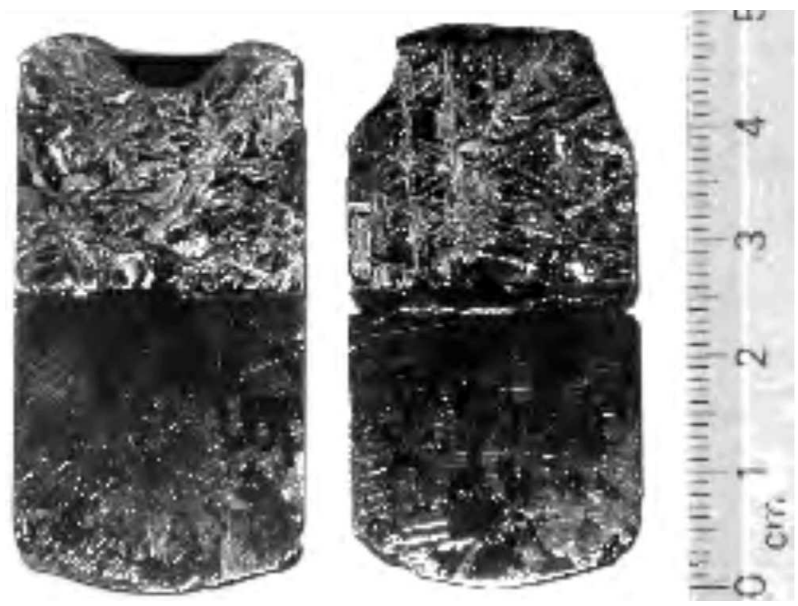

Fig. 20. Samples processed under modified thermal profile.

The EDS composition profiles support the observations from the samples. The EDS data is plotted in Fig. 21. In the region near the bottom of the crucible, the silicon composition is much higher than that observed in any other LPD experiment. The profile rises in silicon concentration from this point to the discontinuity line noted in the etched samples. There is a slight turn up in composition around the bottom of the crucible. This is indicative of self-seeding on the bottom of the crucible due to a lack of seed material. The upturn is due to additional constitutional super-cooling needed to trigger a nucleation process. On the other side of the discontinuity, the melt composition drops to a much lower silicon concentration. The measurements are scattered in this region of the melt due to the segregation. However, there is a rising trend in the silicon composition towards the dissolution interface.

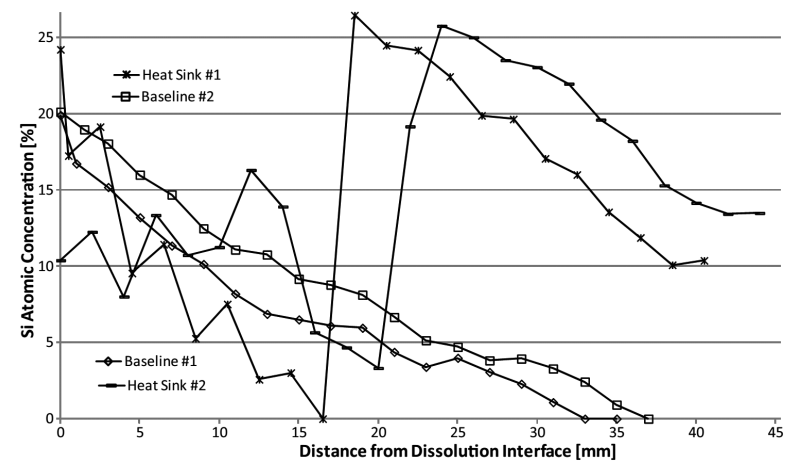

Fig. 21. EDS composition profiles.

The observed profile can be explained as follows. The discontinuity line would seem to indicate that this was the point that growth had reached when the cooling of the furnace was begun at the end of the $72 \mathrm{~h}$ experiment time. The segregation in the solid above the discontinuity is indicative of a quenched melt.

The high silicon region of the material below the discontinuity is considered to have been solidified during the experiment time by the same mechanism as LPD growth. The melt in front of the growth interface is constitutionally super-cooled by transported silicon and solidification takes place on the growth interface. Using the SiGe phase diagram, the liquidus composition values can be calculated for the observed solidified material composition. This indicates the required silicon concentration in the melt for solidification to occur by constitutional super-cooling. Due to the large separation between solidus and liquidus in the SiGe phase diagram, these values are much lower than the solid composition values.

The calculated liquidus composition profiles for the solidified material match the calculated diffusion profile quite well. This is shown in Fig. 22. This indicates that the growth could have been driven by a diffusion-dominated process. The high degree of germanium rejection back into the melt will drive high concentration gradients in the growth interface boundary layer. This should speed transport of silicon from the bulk melt to the interface, allowing growth to proceed quickly. This is additionally helped by lower axial temperature gradients in the melt. The reduced gradient is expected due to the introduced heat from the graphite and it is evidenced from the shallow slope of the liquidus compositions. The flatness of the axial temperature gradient means the saturation composition of the melt increases less quickly as growth proceeds. The primary effect limiting growth 


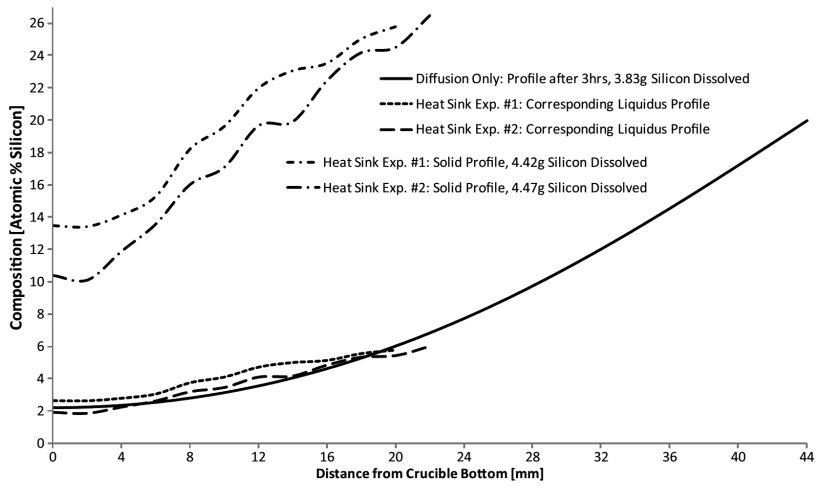

Fig. 22. Calculated liquidus composition profiles.

rate would seem to be the germanium segregated at the growth interface. These factors could allow the growth to proceed faster than seen in other experiments.

The total dissolved silicon incorporated in the solid during the experiment time can be estimated by integrating the composition profile using the discontinuity as the upper bound. Using this method, both samples show similar amounts of incorporated silicon, approximately $4.5 \mathrm{~g}$. This weight is a lower bound on the amount of silicon that must be dissolved during the growth period. The silicon sources used in the experiments weigh approximately $5.7 \mathrm{~g}$. By integrating the calculated diffusion model's compositional profile, the total dissolved silicon in the melt is found to be $3.85 \mathrm{~g}$. Based on this model, the majority of the silicon dissolution occurs during the beginning of the growth process. Given the small difference between the calculated weight of silicon in the grown material and the total weight of the source material, it is possible that growth may have proceeded further if more silicon was available for dissolution. In one of the samples, the silicon source did completely dissolve. In the other, the size of the source was greatly reduced. As the source material dissolves, the surface area in contact with the melt will eventually decrease, limiting the dissolution rate.

\subsection{Remarks}

Once growth is initiated, the majority of the required silicon to produce the observed concentration profile is dissolved in the melt. This will aid in a fast growth rate once growth begins. The dissolution rate can be significantly slower after the initiation of growth and the observed profile achieved. If the dissolution rate does not keep up with the rate of silicon incorporation into the growing solid, the melt will slowly become dilute in silicon and the growth rate will slow. The melt becoming dilute by such a mechanism is likely the cause for the low silicon concentration region observed in the samples. The discontinuity observed between the regions was originally stated as to have been induced by the cooling of the furnace. It is also possible that this was simply the point where the melt became too dilute in silicon to support any further growth.

These experiments show that the LPD technique could be extended to much higher silicon concentrations than those that have been currently produced. Despite the high segregation of silicon, the growth proceeds in a similar manner to previous experiments. The issue of seeds for growth at higher compositions could be overcome by utilizing successive LPD experiments, increasing the temperature between runs. The first run could produce material high enough in composition to be used as seed material for the next run. Higher concentrations could also be achieved with a longer melt zone. In that case, a pure germanium seed could be used. The growth time would have to be extended to allow the silicon to diffuse across the greater distance between the dissolution and growth interfaces. It is predicted that this approach would significantly slow the growth rate at the start of the process, potentially making the technique infeasible.

\section{Conclusions}

Experimental work on the nature of silicon dissolution and transport in a silicon-germanium melt has been conducted for the aim of refining approaches to melt growth seed production and melt replenishment techniques. Experiments performed with and without the application of external magnetic fields have shown that replenishment from bottom of the crucible is most effective due to the enhanced silicon transport by buoyancy. The application of a static magnetic field has altered the thermal characteristics of the dissolution process being not so favorable. However, the application of either a RMF alone or an RMF combined with static field provides better mixing and control of replenishment. Experiments using long capillary samples have shown similar trends.

The LPD technique was also explored as a method of producing seed material and for insight into transport. Experiments with and without the application of external magnetic fields have shown similar observations obtained in the dissolution experiments, namely, an RFM alone or combined with static field is beneficial, but the application of a static field alone disturbs the stable growth mechanism of LPD. Experiments under a modified thermal profile (with heat sink at bottom) provide a better control for obtaining higher silicon compositions (about $20 \%$ ) in the grown crystals. This implies that in our future LPD experiments under appropriate crucible translation, SiGe seed crystals at desired uniform silicon compositions can be obtained.

\section{Acknowledgments}

Financial support provided by the Canadian Space Agency (CSA), the Canada Research Chairs (CRC) Program, and the Natural Sciences and Engineering Research Council (NSERC) of Canada is gratefully acknowledged. 


\section{References}

[1] E. Kasper, J. Cryst. Growth 150, 921 (1995).

[2] I. Yonenaga, J. Cryst. Growth 275, 91 (2005).

[3] N. Abrosimov, S. Rossolenko, V. Alex, A. Gerhardt, W. Schroder, J. Cryst. Growth 166, 657 (1966).

[4] I. Yonenaga, M. Nonaka, J. Cryst. Growth 191, 393 (1998).

[5] X. Niu, W. Zhang, G. Lu, Z. Jiang, J. Cryst. Growth 267, 424 (2004).

[6] N. Usami, R. Nihei, I. Yonenaga, Y. Nose, K. Nakajima, Appl. Phys. Lett. 90, 181914 (2007).

[7] D. Yang, J. Chen, H. Li, X. Ma, D. Tian, L. Li, D. Que, J. Cryst. Growth 292, 266 (2006).

[8] G. Azhdarov, T. Kucukomeroglu, A. Varilci, M. Altunbas, A. Kobya, P. Azhdarov, J. Cryst. Growth 226, 437 (2001).

[9] N. Abrosimov, S. Rossolenko, W. Thieme, A. Gerhardt, W. Schroder, J. Cryst. Growth 174, 182 (1997).

[10] I. Yonenaga, Y. Murakami, J. Cryst. Growth 191, 399 (1998).

[11] A. Matsui, I. Yonenaga, K. Sumino, J. Cryst. Growth 183, 109 (1998).

[12] A. Dahlen, A. Fattah, G. Hanke, E. Karthaus, Cryst. Res. Technol. 29, 187 (1994).

[13] N. Armour, S. Dost, B. Lent, J. Cryst. Growth 299 , 227 (2007).
[14] M. Yildiz, S. Dost, B. Lent, J. Cryst. Growth 280 , 151 (2005).

[15] A. Borshchevsky, J.P. Fleurial, J. Cryst. Growth 128, 331 (1993).

[16] E. Yildiz, S. Dost, M. Yildiz, J. Cryst. Growth 291 , 497 (2006).

[17] E. Yildiz, S. Dost, J. Cryst. Growth 303, 279 (2007).

[18] N. Armour, S. Dost, Fluid Dyn. Mater. Proc. 5, 331 (2009).

[19] F. Micheghel, N. Armour, S. Dost, M. Kadja, TWMS J. Appl. Engr. Math. 1, 127 (2011).

[20] N. Armour, S. Dost, J. Cryst. Growth 306, 200 (2007).

[21] N. Armour, S. Dost, J. Phys., Conf. Series 327, 012016 (2011).

[22] N. Usami, M. Kitamura, K. Obara, Y. Nose, T. Shishido, K. Nakajima, J. Cryst. Growth 284, 57 (2005).

[23] M. Yildiz, S. Dost, B. Lent, Cryst. Res. Technol. 41, 211 (2006).

[24] N. Armour, M. Yildiz, E. Yildiz, S. Dost, ECS Trans. 16, 135 (2008)

[25] N. Armour, S. Dost, Cryst. Res. Technol. 45, 244 (2010).

[26] N. Armour, S. Dost, Cryst. Res. Technol. 45, 335 (2010). 